Bol. Soc. Esp. Mat. Apl.

$n^{\circ}$ 51(2010), 91-99

\title{
THE SUB-SUPERTRAJECTORY METHOD. APPLICATION TO THE NONAUTONOMOUS COMPETITION LOTKA-VOLTERRA MODEL
}

\author{
J.A. LANGA*, A. RODRíGUEZ-BERNAL ${ }^{\ddagger}$ AND A. SUÁREZ ${ }^{\dagger}$ \\ ${ }^{* \dagger}$ Dpto. EDAN, University of Sevilla \\ Aptdo. 1160, 41080 SEVILLA \\ ${ }^{\ddagger}$ Departamento de Matemática Aplicada, \\ Universidad Complutense de Madrid, 28040, \\ Instituto de Ciencias Matemáticas \\ CSIC-UAM-UC3M-UCM, MADRID \\ langa@us.es arober@mat.ucm.es suarez@us.es
}

\begin{abstract}
In this paper we study in detail the pullback and forwards attractions to non-autonomous competition Lotka-Volterra system. In particular, under some conditions on the parameters, we prove the existence of a unique non-degenerate global solution for these models, which attracts any other complete bounded trajectory. For that we present the sub-supertrajectory tool as a generalization of the now classical subsupersolution method.
\end{abstract}

Key words: Sub-supertrajectory method, Lotka-Volterra competition system, attracting complete trajectories.

AMS subject classifications: $\quad 35 B 40,35 K 55,92 D 25,37 \mathrm{~L} 05$.

\section{Introduction}

In this paper we collect some results from [6] and [7] to analyze the asymptotic dynamics of the following non-autonomous Lotka-Volterra competition model

$$
\begin{cases}u_{t}-\Delta u=u(\lambda(t, x)-a(t, x) u-b(t, x) v) & x \in \Omega, t>s \\ v_{t}-\Delta v=v(\mu(t, x)-c(t, x) u-d(t, x) v) & x \in \Omega, t>s \\ u=v=0 & x \in \partial \Omega, t>s \\ u(s)=u_{s}, v(s)=v_{s} . & \end{cases}
$$

\footnotetext{
*Partly supported by grants MTM2008-0088, HF2008-0039 and PHB2006-003PC.

${ }^{\dagger}$ Partly supported by grant MTM2006-07932.

${ }^{\ddagger}$ Partly supported by grants MTM2006-08262, CCG07-UCM/ESP-2393 UCM-CAM Grupo de Investigación CADEDIF and PHB2006-003PC.
} 
Here, $u$ and $v$ represent the population densities of two species within a habitat $\Omega$, a bounded and smooth domain in $\mathbb{R}^{N}, N \geq 1$, which compete in the habitat. $\lambda, \mu$ are the growth rates of the species, $b, c$ are the interaction rates between the species, $a, d$ describe the limiting effects of crowding in each population. We are assuming that $\Omega$ is fully surrounded by inhospitable areas, since the population densities are subject to homogeneous Dirichlet boundary conditions. $u_{s}, v_{s}$ are regular and positive functions which implies that the solution of (1) satisfies $u, v \geq 0$.

In this work we are interested in determining the asymptotic behaviour of solutions of the system (1). This is a very complicated task, and only partial results are known. For example in the autonomous case (all the coefficients in (1) are constants) and denoting by $\Lambda_{0}$ the principal eigenvalue associated to $-\Delta$, then if $\lambda$ or $\mu \leq \Lambda_{0}$, then one of the two species (or both of them) will be driven to extinction. However, there exist two increasing maps $F, G:\left[\Lambda_{0}, \infty\right) \mapsto \mathbb{R}$ such that if

$$
\lambda>G(\mu) \text { and } \mu>F(\lambda),
$$

then (1) is permanent and moreover there exists a positive equilibrium solution (see Cantrell et al. [2] and López-Gómez [9]).

When non-autonomous terms are allowed in the equations, this is usually done under the assumption of periodicity, quasiperiodicity or almost periodicity, and in this case similar results can be obtained to those for autonomous equations (see Hess [4], Hetzer and Shen [5] and references there in).

Cantrell and Cosner [1] assume general non-autonomous terms that are bounded by periodic functions, and using a comparison method give conditions on $\lambda$ and $\mu$ that guarantee that (1) is permanent.

In [6] we show that, under a smallness condition on the coupling coefficients $b c$, if there exists a bounded and bounded away from zero complete trajectories of (1), it is the unique such trajectory, and it also describes the unique pullback and forwards attracting for $(1)$, i.e. $\left(u^{*}, v^{*}\right)$ is a bounded trajectory such that, for any $s \in \mathbb{R}$ and for any positive solution $(u(t, s), v(t, s))$ of (1) defined for $t>s$, one has

$$
\left(u(t, s)-u^{*}(t), v(t, s)-v^{*}(t)\right) \rightarrow(0,0) \quad \text { as } \quad t \rightarrow \infty, \text { or } \quad s \rightarrow-\infty .
$$

In this work (see [7]) we show that this trajectory really exists. To this end we introduce the sub-supertrajectory method as a tool to get existence of intermediate complete trajectories associated to (1). Note that our construction is independent of whether or not (1) has monotonicity properties. Note also that the usual way in previous works (for instance [6], [11]) to get existence of complete trajectories associated to a particular system is by means of the pullback attractor. The sub-supertrajectory method adopts a different and, in this case, more fruitful strategy. Moreover, we also get the existence of minimal and maximal global bounded trajectories associated to ordered systems.

In Section 2 we present the sub-supertrajectory tool, Section 3 is devoted to the logistic equation which appears when one species is absent. Finally, in Section 4 we show the results of system (1). 


\section{The sub-supertrajectory method for complete solutions}

Consider the general problem

$$
\begin{cases}u_{t}-\Delta u=f(t, x, u, v) & x \in \Omega, t>s \\ v_{t}-\Delta v=g(t, x, u, v) & x \in \Omega, t>s \\ u=v=0 & x \in \partial \Omega, t>s \\ u(s)=u_{s}, v(s)=v_{s}, & \end{cases}
$$

where $f, g$ are bounded on bounded sets of $\mathbb{R} \times \bar{\Omega} \times \mathbb{R}^{2}$ and are locally Hölder continuous in time. We denote the solutions of (3) as

$$
u\left(t, s ; u_{s}, v_{s}\right), \quad v\left(t, s ; u_{s}, v_{s}\right), \quad \text { for } t>s .
$$

Definition 1 A pair of functions $(u, v) \in C_{t, x}^{1,2}(\mathbb{R} \times \bar{\Omega})$ is a complete trajectory of (3), if for all $s<t$ in $\mathbb{R},(u(t), v(t))$ is the solution of (3) with initial data $u_{s}=u(s), v_{s}=v(s)$.

Definition 2 A positive function $u(t, x)$ is non-degenerate at $\infty$ (respectively $-\infty)$ if there exists $t_{0} \in \mathbb{R}$ such that $u$ is defined in $\left[t_{0}, \infty\right.$ ) (respectively $\left.\left(-\infty, t_{0}\right]\right)$ and there exists a $C_{0}^{1}(\bar{\Omega})$ function $\varphi_{0}(x)>0$ in $\Omega$, such that for all $x \in \bar{\Omega}, u(t, x) \geq \varphi_{0}(x)$ for all $t \geq t_{0}$ (respectively for all $t \leq t_{0}$ ).

The use of sub-supertrajectory pairs to construct complete solutions can be found in Chueshov [3] or Langa and Suárez [8]. Both references use monotonicity properties of the equations, see Corollaries 2 and 3 below. In particular this applies to scalar equations. Here we use similar ideas to construct bounded complete trajectories, without such monotonicity assumptions.

Given $T_{0} \leq \infty$ and two functions $w, z \in C\left(\left(-\infty, T_{0}\right) \times \bar{\Omega}\right)$ with $w \leq z$ we denote

$$
[w, z]:=\left\{u \in C\left(\left(-\infty, T_{0}\right) \times \bar{\Omega}\right): w \leq u \leq z\right\} .
$$

Now we introduce the concept of complete sub-supertrajectory pair.

Definition 3 Let $T_{0} \leq \infty$ and $(\underline{u}, \underline{v}),(\bar{u}, \bar{v}) \in \mathcal{X}=C_{t, x}^{1,2}\left(\left(-\infty, T_{0}\right) \times \bar{\Omega}\right)$. We say that $(\underline{u}, \underline{v})-(\bar{u}, \bar{v})$ is a complete sub-supertrajectory pair of (3) if

1. $\underline{u}(t) \leq \bar{u}(t)$ and $\underline{v}(t) \leq \bar{v}(t)$ in $\Omega$, for all $t<T_{0}$.

2. $\underline{u} \leq 0 \leq \bar{u}$ and $\underline{v} \leq 0 \leq \bar{v}$ on $\partial \Omega$, for all $t<T_{0}$.

3. For all $x \in \Omega, t<T_{0}$

$$
\begin{aligned}
& \underline{u}_{t}-\Delta \underline{u}-f(t, x, \underline{u}, v) \leq 0 \leq \bar{u}_{t}-\Delta \bar{u}-f(t, x, \bar{u}, v), \quad \forall v \in[\underline{v}, \bar{v}], \\
& \underline{v}_{t}-\Delta \underline{v}-g(t, x, u, \underline{v}) \leq 0 \leq \bar{v}_{t}-\Delta \bar{v}-g(t, x, u, \bar{v}), \quad \forall u \in[\underline{u}, \bar{u}] .
\end{aligned}
$$

Note that the concept of a sub-supersolution pair, defined for $t>s$, has been widely used and developed, see e.g. Pao [10], to construct solutions for the initial value problem (3). The main result of this section is: 
Theorem 1 Assume that there exists a complete sub-supertrajectory pair of (3), $(\underline{u}, \underline{v})-(\bar{u}, \bar{v})$, in the sense of Definition 3. Moreover, assume $\underline{u}, \underline{v}, \bar{u}$ and $\bar{v}$ are bounded at $-\infty$. Then, there exists a complete trajectory $\left(u^{*}, v^{*}\right) \in \mathcal{X}$ of (3) such that

$$
\left(u^{*}, v^{*}\right) \in \mathcal{I}:=[\underline{u}, \bar{u}] \times[\underline{v}, \bar{v}] .
$$

When $f$ and $g$ have some monotonicity properties, we can go further:

Corollary 2 Under the assumptions of Theorem 1, assume moreover that $f$ is increasing in $v$ and $g$ in $u$. Then, there exist two complete trajectories $\left(u_{*}, v_{*}\right)$ and $\left(u^{*}, v^{*}\right)$ of (3) with $\left(u_{*}, v_{*}\right),\left(u^{*}, v^{*}\right) \in \mathcal{I}:=[\underline{u}, \bar{u}] \times[\underline{v}, \bar{v}]$ such that they are minimal and maximal in $\mathcal{I}$ in the following sense: for any other complete trajectory $(u, v) \in \mathcal{I}$ we have:

$$
\begin{aligned}
& \underline{u}(t) \leq u_{*}(t) \leq u(t) \leq u^{*}(t) \leq \bar{u}(t), \quad \text { for all } t<T_{0} . \\
& \underline{v}(t) \leq v_{*}(t) \leq v(t) \leq v^{*}(t) \leq \bar{v}(t), \quad \text {. }
\end{aligned}
$$

Corollary 3 Under the assumptions of Theorem 1, assume moreover that $f$ is decreasing in $v$ and $g$ in $u$. Then, there exist two complete trajectories $\left(u_{*}, v^{*}\right)$ and $\left(u^{*}, v_{*}\right)$ of (3) with $\left(u_{*}, v^{*}\right),\left(u^{*}, v_{*}\right) \in \mathcal{I}:=[\underline{u}, \bar{u}] \times[\underline{v}, \bar{v}]$ and such that they are minimal-maximal and maximal-minimal in the following sense: for any other complete trajectory $(u, v) \in \mathcal{I}$ we have:

$$
\begin{aligned}
& \underline{u}(t) \leq u_{*}(t) \leq u(t) \leq u^{*}(t) \leq \bar{u}(t), \quad \text { for all } t<T_{0} . \\
& \underline{v}(t) \leq v_{*}(t) \leq v(t) \leq v^{*}(t) \leq \bar{v}(t), \quad
\end{aligned}
$$

\section{The non-autonomous logistic equation}

Note that (1) always admits semi-trivial trajectories of the form $(u, 0)$ or $(0, v)$. In this case, when one species is not present, the other one satisfies the logistic equation

$$
\begin{cases}u_{t}-\Delta u=h(t, x) u-g(t, x) u^{2} & \text { in } \Omega, t>s \\ u=0 & \text { on } \partial \Omega, \\ u(s)=u_{s} \geq 0 & \text { in } \Omega .\end{cases}
$$

It is well known that if

$$
h_{M}:=\sup _{\bar{Q}} h(t, x)<\infty \quad \text { and } \quad g_{L}:=\inf _{\bar{Q}} g(t, x)>0,
$$

then, for every non-trivial $u_{s} \in C(\bar{\Omega}), u_{s} \geq 0$, there exists a unique positive solution of $(6)$ denoted by $\Theta_{[h, g]}\left(t, s ; u_{s}\right)$.

On the other hand, for $m \in L^{\infty}(\Omega)$ we denote by $\Lambda(m)$, the first eigenvalue of

$$
-\Delta u=\lambda u+m(x) u \quad \text { in } \Omega, \quad u=0 \quad \text { on } \partial \Omega .
$$

In particular, we denote by $\Lambda_{0}:=\Lambda(0)$. It is well known that $\Lambda(m)$ is a simple eigenvalue with a positive eigenfunction, and a continuous and decreasing function of $m$. 
Finally, for $h, g \in L^{\infty}(\Omega)$ with $g_{L}:=\inf \{g(x), x \in \bar{\Omega}\}>0$ consider the elliptic equation

$$
\begin{cases}-\Delta u=h(x) u-g(x) u^{2} & \text { in } \Omega, \\ u=0 & \text { on } \partial \Omega .\end{cases}
$$

It is well known that (8) possesses a unique positive solution if, and only if, $\Lambda(h)<0$, which we denote by $\omega_{[h, g]}(x)$.

In the following result (see [12], [11] and [7] for a complete study of (6)) we show the existence and properties of a complete nonnegative trajectory for (6). For this we will assume henceforth that $h(t, x)$ and $g(t, x)$ satisfy $(7)$ and there exist bounded functions $h_{0}^{ \pm}(x)$ and $H_{0}^{ \pm}(x)$ defined in $\Omega$ such that

$$
\limsup _{t \rightarrow \pm \infty} \sup _{x \in \Omega}\left(h(t, x)-H_{0}^{ \pm}(x)\right) \leq 0, \quad 0 \leq \liminf _{t \rightarrow \pm \infty} \inf _{x \in \Omega}\left(h(t, x)-h_{0}^{ \pm}(x)\right) .
$$

Proposition 4 Assume (7) and (9). Then:

i) There exists a maximal bounded complete trajectory, denoted by $\varphi_{[h, g]}(t)$, of $(6)$, in the sense that, for any other non-negative complete bounded trajectory $\xi(t)$ of (6) we have

$$
0 \leq \xi(t) \leq \varphi_{[h, g]}(t), \quad t \in \mathbb{R} .
$$

Moreover, if $\varphi_{[h, g]}(t, x)$ is nondegenerate at $-\infty$ then it is the only one of such solutions.

ii) If $\Lambda\left(H_{0}^{-}\right)>0$, then $\varphi_{[h, g]}(t)=0$ for all $t \in \mathbb{R}$. Therefore all non-negative solutions of (6) converge to 0 , uniformly in $\Omega$, in the pullback sense.

iii) If $\Lambda\left(h_{0}^{-}\right)<0$ then $\varphi_{[h, g]}$ is the unique complete bounded and non-degenerate trajectory at $-\infty$ of (6), and for $t$ in compact sets of $\mathbb{R}$, if $s \mapsto u_{s} \geq 0$ is bounded and non-degenerate, then

$$
\Theta_{[h, g]}\left(t, s ; u_{s}\right)-\varphi_{[h, g]}(t) \rightarrow 0 \quad \text { as } s \rightarrow-\infty \quad \text { uniformly in } \Omega .
$$

iv) If $\Lambda\left(H_{0}^{+}\right)>0$, then for all $u_{s} \in C(\bar{\Omega}), u_{s} \geq 0$, the positive solution of (6) satisfies $\Theta_{[h, g]}\left(t, s ; u_{s}\right) \rightarrow 0$ uniformly in $\Omega$ as $t \rightarrow \infty$. In particular, $\varphi_{[h, g]}(t) \rightarrow 0$ uniformly in $\Omega$ as $t \rightarrow \infty$.

v) If $\Lambda\left(h_{0}^{+}\right)<0$ and $\varphi_{[h, g]} \neq 0$, then $\varphi_{[h, g]}$ is non-degenerate at $\infty$ and for any $s$ and any non-trivial initial data $u_{s} \geq 0$,

$$
\Theta_{[h, g]}\left(t, s ; u_{s}\right)-\varphi_{[h, g]}(t) \rightarrow 0 \quad \text { in } C^{1}(\bar{\Omega}) \text { as } t \rightarrow \infty .
$$

\section{Applications to the Lotka-Volterra competition model}

We assume from now on that $\lambda, \mu \in \mathbb{R}$ and

$$
a_{L}, d_{L}, b_{L}, c_{L}>0
$$

We will assume that there exist quantities $a_{I}^{ \pm} \leq a_{S}^{ \pm}, b_{I}^{ \pm} \leq b_{S}^{ \pm}, c_{I}^{ \pm} \leq c_{S}^{ \pm}$and $d_{I}^{ \pm} \leq d_{S}^{ \pm}$such that

$$
\begin{array}{ll}
0<a_{I}^{ \pm} \leq a(t, x) \leq a_{S}^{ \pm}, & 0<b_{I}^{ \pm} \leq b(t, x) \leq b_{S}^{ \pm}, \\
0<c_{I}^{ \pm} \leq c(t, x) \leq c_{S}^{ \pm}, & 0<d_{I}^{ \pm} \leq d(t, x) \leq d_{S}^{ \pm},
\end{array}
$$


for all $x \in \Omega$ and for all $t \geq t_{0}$ or $t \leq t_{0}$. In the following result we show the existence of a complete trajectory of (1).

Proposition 5 (Competitive case) There exists a complete trajectory $\left(u^{*}, v^{*}\right)$ of (1) with

$\varphi_{\left[\lambda-b \varphi_{[\mu, d]}, a\right]}(t) \leq u^{*}(t) \leq \varphi_{[\lambda, a]}(t), \varphi_{\left[\mu-c \varphi_{[\lambda, a]}, d\right]}(t) \leq v^{*}(t) \leq \varphi_{[\mu, d]}(t), \quad t \in \mathbb{R}$.

Moreover, if (11) is satisfied for very negative $t$ and

$$
\lambda>\Lambda\left(-b_{S}^{-} \omega_{\left[\mu, d_{I}^{-}\right]}\right) \quad \text { and } \quad \mu>\Lambda\left(-c_{S}^{-} \omega_{\left[\lambda, a_{I}^{-}\right]}\right),
$$

then $\left(u^{*}, v^{*}\right)$ is non-degenerate at $-\infty$.

If moreover (11) is satisfied for large and very negative $t$, (12) and

$$
\lambda>\Lambda\left(-b_{S}^{+} \omega_{\left[\mu, d_{I}^{+}\right]}\right) \quad \text { and } \quad \mu>\Lambda\left(-c_{S}^{+} \omega_{\left[\lambda, a_{I}^{+}\right]}\right)
$$

holds, then $\left(u^{*}, v^{*}\right)$ is non-degenerate at $\infty$.

Proof. Note that in this case $f$ is decreasing in $v$ and $g$ in $u$. It is enough to take

$$
(\underline{u}, \bar{u})=\left(\varphi_{\left[\lambda-b \varphi_{[\mu, d]}, a\right]}, \varphi_{[\lambda, a]}\right) \quad \text { and } \quad(\underline{v}, \bar{v})=\left(\varphi_{\left[\mu-c \varphi_{[\lambda, a]}, d\right]}, \varphi_{[\mu, d]}\right) .
$$

Moreover, if $\lambda$ and $\mu$ satisfy (12), resp. (13), then by Proposition 6 we obtain that $\underline{u}$ and $\underline{v}$ are non-degenerate at $-\infty$, resp. $+\infty$.

Now, we can summarize the results for the system (1).

\section{Theorem 6 (Competitive case)}

1. If $\lambda<\Lambda_{0}$ and $\mu<\Lambda_{0}$

$$
\lim _{s \rightarrow-\infty}\left(u\left(t, s ; u_{s}, v_{s}\right), v\left(t, s ; u_{s}, v_{s}\right)\right)=\lim _{t \rightarrow \infty}\left(u\left(t, s ; u_{s}, v_{s}\right), v\left(t, s ; u_{s}, v_{s}\right)\right)=(0,0) .
$$

2. If $\lambda<\Lambda_{0}$ and $\mu>\Lambda_{0}$, then

$$
\lim _{t \rightarrow \infty} u\left(t, s ; u_{s}, v_{s}\right)=0,
$$

and for every nonnegative nontrivial $\tilde{v}_{s}$ we have

$\lim _{t \rightarrow \infty}\left(v\left(t, s ; u_{s}, v_{s}\right)-\Theta_{[\mu, d]}\left(t, s ; \tilde{v}_{s}\right)\right)=\lim _{t \rightarrow \infty}\left(v\left(t, s ; u_{s}, v_{s}\right)-\varphi_{[\mu, d]}(t)\right)=0$.

3. If $\lambda>\Lambda_{0}$ and $\mu<\Lambda_{0}$, then

$$
\lim _{t \rightarrow \infty} v\left(t, s ; u_{s}, v_{s}\right)=0,
$$

and for every nonnegative nontrivial $\tilde{v}_{s}$ we have

$$
\lim _{t \rightarrow \infty}\left(u\left(t, s ; u_{s}, v_{s}\right)-\Theta_{[\lambda, a]}\left(t, s ; \tilde{v}_{s}\right)\right)=\lim _{t \rightarrow \infty}\left(u\left(t, s ; u_{s}, v_{s}\right)-\varphi_{[\lambda, a]}(t)\right)=0 .
$$


4. If

$$
\lambda>\Lambda\left(-b_{S}^{-} \omega_{\left[\mu, d_{I}^{-}\right]}\right) \quad \text { and } \quad \mu>\Lambda\left(-c_{S}^{-} \omega_{\left[\lambda, a_{I}^{-}\right]}\right),
$$

there exists a complete bounded non-degenerate at $-\infty$ trajectory of (1) $\left(u^{*}(t), v^{*}(t)\right)$. Moreover, if $b$ or $c$ are small at $-\infty$, that is,

$$
\limsup _{t \rightarrow-\infty}\|b\|_{L^{\infty}(\Omega)} \limsup _{t \rightarrow-\infty}\|c\|_{L^{\infty}(\Omega)}<\rho_{0}
$$

for some suitable constant $\rho_{0}>0$, then this is the unique bounded nondegenerate at $-\infty$ trajectory of (1) and it is pullback attracting, that is

$$
\lim _{s \rightarrow-\infty}\left(u\left(t, s ; u_{s}, v_{s}\right)-u^{*}(s), v\left(t, s ; u_{s}, v_{s}\right)-v^{*}(s)\right)=(0,0) .
$$

If moreover

$$
\lambda>\Lambda\left(-b_{S}^{+} \omega_{\left[\mu, d_{I}^{+}\right]}\right) \quad \text { and } \quad \mu>\Lambda\left(-c_{S}^{+} \omega_{\left[\lambda, a_{I}^{+}\right]}\right),
$$

then $\left(u\left(t, s ; u_{s}, v_{s}\right), v\left(t, s ; u_{s}, v_{s}\right)\right)$ is non-degenerate at $\infty$. If additionally $b$ or $c$ are small at $\infty$, that is,

$$
\limsup _{t \rightarrow \infty}\|b\|_{L^{\infty}(\Omega)} \limsup _{t \rightarrow \infty}\|c\|_{L^{\infty}(\Omega)}<\rho_{0}
$$

for some suitable constant $\rho_{0}>0$, then all solutions of (1) have the same asymptotic behavior as $t \rightarrow \infty$. If (14) is also satisfied, then $\left(u^{*}(t), v^{*}(t)\right)$ is non-degenerate at $\infty$ and it is also forwards attracting, that is,

$$
\lim _{t \rightarrow \infty}\left(u\left(t, s ; u_{s}, v_{s}\right)-u^{*}(t), v\left(t, s ; u_{s}, v_{s}\right)-v^{*}(t)\right)=(0,0) .
$$

Remark 1 Similar results can be presented for the prey-predator and symbiosis cases.

In Figure 1 we describe the asymptotic dynamical regimes (pullback -Case a)- and forwards -Case b)) when $\lambda$ and $\mu$ are constant functions. Region A: extinction of both species; Regions B and C: stability of semitrivial complete trajectories; Regions $D_{P}$ and $D_{F}$ : permanence regions (existence of global nondegenerate global solutions). The limiting curves are given in (14) and (15).

\section{References}

[1] R. S. Cantrell and C. Cosner, Practical persistence in ecological models via comparison methods, Proc. Royal Soc. Edin., 126A (1996) 247-272.

[2] R. S. Cantrell and C. Cosner, Spatial Ecology via Reaction-Diffusion Equations, John Wiley \& Sons. Ltd. 2003.

[3] I. Chueshov, Monotone random systems theory and applications. Lecture Notes in Mathematics, 1779. Springer-Verlag, Berlin, 2002. 


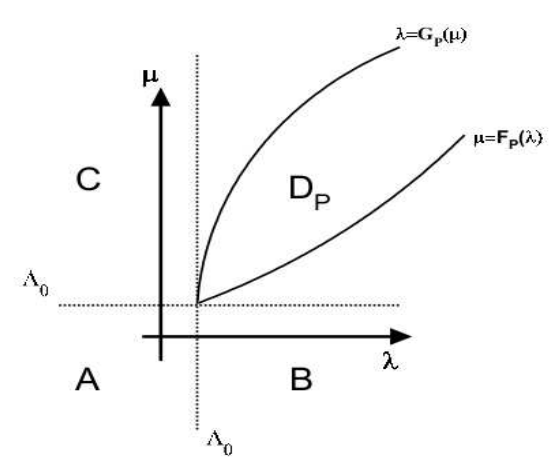

Case a)

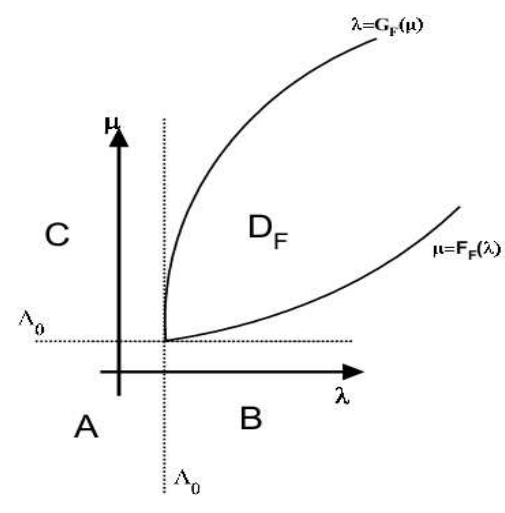

Case b)

[4] P. Hess, Periodic-Parabolic boundary value problems and positivity, Pitman Research Notes in Mathematics 247, Harlow Longman. 1991.

[5] G. Hetzer, W. Shen, Uniform persistence, coexistence, and extinction in almost periodic/nonautonomous competition diffusion systems, SIAM J. Math. Anal., 34 (2002) 204-221.

[6] J. A. Langa, J.C. Robinson, A. Rodríguez-Bernal and A. Suárez, Permanence and asymptotically stable complete trajectories for nonautonomous Lotka-Volterra models with diffusion, SIAM J. Math. Anal. 40 (2009) 2179-2216.

[7] J. A. Langa, A. Rodríguez-Bernal and A. Suárez, On the long time behaviour of non-autonomous Lotka-Volterra models with diffusion via the sub-super trajectory method, submitted.

[8] J. A. Langa and A. Suárez, Pullback permanence for non-autonomous partial differential equations, Electron. J. Differential Equations 2002, 72, 20 pp.

[9] J. López-Gómez, On the structure of the permanence region for competing species models with general diffusivities and transport effects,Discrete Contin. Dyn. Syst., 2 (1996) 525-542.

[10] C. V. Pao, Nonlinear parabolic and elliptic equations, Plenum, New York, 1992.

[11] J.C. Robinson, A. Rodríguez-Bernal, and A. Vidal-López, Pullback attractors and extremal complete trajectories for non-autonomous reactiondiffusion problems, J. Differential Equations 238 (2007) 289-337.

[12] A. Rodríguez-Bernal and A. Vidal-López, Existence, uniqueness and attractivity properties of positive complete trajectories for non-autonomous reaction-diffusion problems, Discrete Contin. Dyn. Syst., 18 (2007) 537-567. 